\title{
Starting the Dismantling of Classical Mathematics
}

\author{
Ross T. Brady \\ La Trobe University \\ Melbourne, Australia \\ Ross.Brady@latrobe.edu.au
}

Dedicated to Richard Routley/Sylvan, on the occasion of the $20^{t h}$
anniversary of his untimely death.

\section{Introduction}

Richard Sylvan (né Routley) has been the greatest influence on my career in logic. We met at the University of New England in 1966, when I was a Master's student and he was one of my lecturers in the M.A. course in Formal Logic. He was an inspirational leader, who thought his own thoughts and was not afraid to speak his mind. I hold him in the highest regard. He was very critical of the standard Anglo-American way of doing logic, the so-called classical logic, which can be seen in everything he wrote. One of his many critical comments was: "Gödel's (First) Theorem would not be provable using a decent logic". This contribution, written to honour him and his works, will examine this point among some others.

Hilbert referred to non-constructive set theory based on classical logic as "Cantor's paradise". In this historical setting, the constructive logic and mathematics concerned was that of intuitionism. (The Preface of Mendelson [2010] refers to this.) We wish to start the process of dismantling this classical paradise, and more generally classical mathematics. Our starting point will be the various diagonal-style arguments, where we examine whether the Law of Excluded Middle (LEM) is implicitly used in carrying them out. This will include the proof of Gödel's First Theorem, and also the proof of the undecidability of Turing's Halting Problem. In the process, we compare these with paradox derivation and Cantor's diagonal argument. The core of mathematics may well be re-constructible to a large extent without the LEM as a general logical principle, in a manner set out, for example, in Brady [2012] for Peano arithmetic, but we will argue here that these diagonal arguments would stand out as being illicit in such a process.

Our logic MC of meaning containment is constructive in a different sense to that of intuitionist logic. (See Brady [2006] and Brady and Meinander [2013] for the motivation and presentation of MC and its quantified extension 
MCQ, and see below for their axiomatizations. ${ }^{1}$ ) The detailed differences in constructivity are set out in Brady [201x]. The main point of difference is that MC embraces meaning containment in a logic of proof, whereas intuitionist logic is also a logic of proof but maintains much more inferential classical logic in the process. Importantly, in keeping with intuitionist logic, $\mathrm{MC}$ rejects the LEM, but $\mathrm{MC}$ differs from intuitionist logic in that it also rejects $A \rightarrow \sim A \rightarrow \sim A$, which is used in paradox derivation in intuitionist logic. An important point of difference is that MC, unlike intuitionist logic, contains all the laws of contraposition as well as those of double negation, which then yields all the De Morgan laws. In our dismantling of classical mathematics, the re-construction would be done in terms of MC and MCQ, which is well-motivated in Brady [2006] and Brady and Meinander [2013], together with Brady [2015] which paints the big picture.

Another form of constructivity in logic is due to Nelson, whose logics can be found in Odintsov [2008] and in Kamide and Wansing [2015]. He introduced a notion of constructible falsity centring on the properties:

If $\sim(A \& B)$ is provable then either $\sim A$ is provable or $\sim B$ is provable.

If $\sim \forall x A$ is provable then so is $\sim A t / x$, for some term $t$.

Both of these also hold for MCQ, with the use of the Priming and Existential Properties, together with De Morgan properties for \& and $\forall$ (see below). However, Nelson's logics differ from MCQ in that contraposition fails in the form $A \rightarrow B \rightarrow \sim B \rightarrow \sim A$, and his logics would lead to a different analysis of the relationship between reductio arguments and the LEM than that carried out in $\S 2$ below.

The theme running through this paper is the use of the LEM, not only in the derivation of the set-theoretic and semantic paradoxes, but also in the extension of its use to the three key diagonal-style arguments of mathematics: Cantor's, Gödel's and Turing's. (See Brady [2015a] for the solution of the paradoxes using the logic MC to capture definitions as meaning equivalences, and Brady [201x] for discussion of the LEM.) Thus, the rejection of the LEM in the logic MC transmits to these diagonal arguments, the removal of which would then require a major re-think to assess the consequences, which we will initiate in $\S 7$.

Moreover, Cantor's diagonal argument and consequent theorem have already been dealt with in Brady and Rush [2008]. We proceed by looking into further diagonal-style arguments in the derivation of Gödel's Theorem, as in Mendelson [2010], and also in the derivation of the undecidability of Turing's halting problem, as derived in Papadimitriou [1994]. The idea of doing this was first mentioned in the conclusion of the paper, Brady [2015], as a possible subject of further study, the beginnings of which will be attempted in this paper.

For future reference, we present the logic MC and its quantificational 
extension MCQ as follows, using the bracketing convention from Anderson and Belnap [1975]:

\section{MC}

Primitives: $\sim, \&, \vee, \rightarrow$

\section{Axioms}

1. $A \rightarrow A$

2. $A \& B \rightarrow A$

3. $A \& B \rightarrow B$

4. $(A \rightarrow B) \&(A \rightarrow C) \rightarrow . A \rightarrow B \& C$

5. $A \rightarrow A \vee B$

6. $B \rightarrow A \vee B$

7. $(A \rightarrow C) \&(B \rightarrow C) \rightarrow . A \vee B \rightarrow C$

8. $\sim A \rightarrow A$

9. $A \rightarrow \sim B \rightarrow . B \rightarrow \sim A$

10. $(A \rightarrow B) \&(B \rightarrow C) \rightarrow . A \rightarrow C$

\section{Rules}

1. $A, A \rightarrow B \Rightarrow B$

2. $A, B \Rightarrow A \& B$

3. $A \rightarrow B, C \rightarrow D \Rightarrow B \rightarrow C \rightarrow . A \rightarrow D$

\section{Meta-Rule.}

1. If $A, B \Rightarrow C$ then $D \vee A, D \vee B \Rightarrow D \vee C$.

We add in the logic $\mathrm{DJ}^{d}$, mentioned in Footnote 1, the logic B, mentioned in $\S 2$, and LDW, mentioned in $\S 7$.

$\mathrm{DJ}^{d}=\mathrm{MC}+$ the distribution axiom:

11. $A \&(B \vee C) \rightarrow(A \& B) \vee(A \& C)$ 
$\mathrm{LDW}=\mathrm{MC}-\mathrm{A} 10-\mathrm{MR} 1$.

$\mathrm{B}=\mathrm{LDW}+\mathrm{A} 11-\mathrm{A} 9+$ the rule-form of A9:

4. $A \rightarrow \sim B \Rightarrow B \rightarrow \sim A$.

We note that distribution in the rule-form, $A \&(B \vee C) \Rightarrow(A \& B) \vee(A \& C)$, is derivable in MC using the meta-rule MR1. The logic MC is a weak relevant logic that is metacomplete ${ }^{2}$, and hence it satisfies the Priming Property (if $A \vee B$ is a theorem, so is either $A$ or $B$ ), often called the Disjunction Property, and is thus disjunctively constructive. $\mathrm{MC}$ is also paranormal (or paraclassical), in that neither the Law of Excluded Middle $(A \vee \sim A)$ nor the Disjunctive Syllogism ( $\sim A, A \vee B \Rightarrow B$ ) (abbreviated as the DS), are derivable within the logic.

We extend the logic MC to MCQ with the following quantificational additions.

\section{MCQ}

Primitives: $\forall, \exists$.

$a, b, c, \ldots$ range over free variables.

$x, y, z, \ldots$ range over bound variables.

Terms $s, t, u, \ldots$ can be individual constants (when introduced) or free variables.

\section{Quantificational Axioms:}

1. $\forall x A \rightarrow A t / x$, for any term $t$

2. $\forall x(A \rightarrow B) \rightarrow . A \rightarrow \forall x B$

3. At/x $\rightarrow \exists x A$, for any term $t$

4. $\forall x(A \rightarrow B) \rightarrow . \exists x A \rightarrow B$

\section{Quantificational Rule:}

1. $A a / x \Rightarrow \forall x A$, where a is not free in $A$

\section{Meta-Rule:}

1. If $A, B a / x \Rightarrow C a / x$ then $A, \exists x B \Rightarrow \exists x C$, where the quantified rule QR1 is not used to generalize on any free variables occurring in the $A$ or in the $B a / x$ of the rule $A, B a / x \Rightarrow C a / x$. This restriction on QR 1 also applies to the rule $A, B \Rightarrow C$ of the meta-rule MR1 for the sentential component.

Note that the existential distribution rule, $A \& \exists x B \Rightarrow \exists x(A \& B)$, follows from R2 and the quantified meta-rule QMR1. However, as with intuitionist logic, the universal distribution rule, $\forall x(A \vee B) \Rightarrow A \vee \forall x B$, fails, 
as the universal quantifier and the disjunction are essentially the same for intuitionist logic as for MCQ, since they are both constructively interpreted concepts. Note also that, being metacomplete, the Existential Property (if $\exists x A$ is a theorem then so is $A t / x$, for some term $t$ ) holds, making the logic existentially constructive which, together with the above Priming Property completes the constructive characterization of the logic MCQ.

There are other proof-theoretic presentations of MCQ, the main one being the natural deduction system in Brady [1984] for $\mathrm{DJ}^{d} \mathrm{Q}$, but with the distribution rules \& $\vee, \forall \vee$ and $\exists \&$ replaced by two-premise versions of $\vee E^{r}$ and $\exists \mathrm{E}^{r}$ to match the two meta-rules MR1 and QMR1. There is also a Gentzen system for DJ in Brady [2006], pp.131-140, and a quantificational extension presented in Brady [2003], pp.350-1.

\section{Reductio Arguments and the LEM}

We start by considering reductio arguments generally, upon which the diagonal arguments are based. As argued in Brady [2015], it can be easily seen that there are two kinds of reductio argument, one involving the LEM and one without using the LEM, though they are both based on contraposition.

First, we consider the derivational form of reductio argument: If $A \Rightarrow$ $B \& \sim B$ then $\sim A$, which is framed as a meta-rule. If we replace the rule $A \Rightarrow B \& \sim B$ by its entailment $A \rightarrow B \& \sim B$ then the reductio argument takes the form: $A \rightarrow B \& \sim B \Rightarrow \sim A$, which is a simple rule based on an entailment. This entailment, $A \rightarrow B \& \sim B$, can be contraposed to obtain $\sim(B \& \sim B) \rightarrow \sim A$, and, by De Morgan's Law and double negation, $(B \vee \sim B) \rightarrow \sim A$. Thus, by the LEM, $\sim A$ is derived. (Indeed, this rule-form, $A \rightarrow B \& \sim B \Rightarrow \sim A$, of reductio is easily seen to be deductively equivalent ${ }^{3}$ to the LEM, given a basic system such as B or MC.) However, the meta-rule is more complicated, due to the use of the rule form of contraposition: If $C \Rightarrow D$ then $\sim D \Rightarrow \sim C$, replacing the entailment form. This can be derived as follows using the LEM for $\mathrm{C}$ and the DS for D. Let $C \Rightarrow D$. Then, by MR1, $\sim C \vee C \Rightarrow \sim C \vee D$ and hence $\sim C \vee D$. Let $\sim D$. Then, by the DS, $\sim C$ follows. Hence, for our derivational form of reductio argument, not only is the LEM applied to $B$, but also $A$, whilst the DS is applied to $B \& \sim B$. Thus, we can see that the LEM plays a key role in deriving these reductio arguments.

The use of the LEM and the DS in deriving the rule form of contraposition can be seen more clearly by inserting $\sim C \vee D$ between $C \Rightarrow D$ and $\sim D \Rightarrow \sim C$, thereby breaking rule contraposition up into the two meta-rule inferences:

If $C \Rightarrow D$ then $\sim C \vee D$ is a theorem.

If $\sim C \vee D$ is a theorem then $\sim D \Rightarrow \sim C$. 
The first is the classical Deduction Theorem, seen to be deductively equivalent to the LEM in the form $\sim C \vee C$, using MR1. The second is an instance of the DS, applied to D.

Let us consider diagonal arguments which use the form: If $A \Rightarrow B \leftrightarrow \sim B$ then $\sim A$, with $B \leftrightarrow \sim B$ replacing $B \& \sim B$, as will be seen in $\S 4$ and $\S 6$ below when our specific diagonal arguments will be considered. This conclusion, $B \leftrightarrow \sim B$, uses a further instance of the LEM in obtaining $B \& \sim B$ to create a derivational reductio argument, as can be seen from its deductive equivalent, $B \vee \sim B \rightarrow B \& \sim B .{ }^{4}$ So, diagonal arguments of this form are essentially reductio arguments, given a usage of the LEM.

These reductio arguments should be contrasted with forms of the sort, $A \rightarrow B, \sim B \Rightarrow \sim A$, which are easily derivable using contraposition, without reference to the LEM. However, the meta-rule-form, If $A \Rightarrow B$ and $\sim B$ then $\sim A$, does use the LEM (as well as the DS), as was shown above for the rule-form of contraposition.

We now briefly examine the LEM itself, a fuller discussion of which can be found in Brady [201x]. As can be seen from its deductive equivalent in MC, $A \rightarrow \sim A \Rightarrow \sim A$ (see Footnote 4 re its derivation), the LEM, $A \vee \sim A$, is basically a form of contraction, which together with other forms of contraction such as $A \rightarrow . A \rightarrow B \Rightarrow A \rightarrow B$, are not derivable in the logic MC. ${ }^{5}$ Also, as above, the rule form of reductio, $A \rightarrow B \& \sim B \Rightarrow \sim A$, is deductively equivalent to the LEM. Thus, these reductio arguments involving the LEM can be thought of as examples of contraction that are not derivable in MC, through such a use of the LEM. However, reductio arguments taking just the form of a contraposition, without the use of the LEM, are included in the logic. As will be shown in $\S 4$ and $\S 6$ below, the diagonal arguments of Cantor and Turing are both examples of reductio arguments of the sort that makes use of the LEM, which is not derivable in MC. Some use is also made of the DS, which is also not derivable in MCQ. As will be seen, the diagonal argument of Gödel is immersed in overall reductio arguments of a more complex kind.

Brady $[201 x]$ argues against the general use of the LEM in that this would include usage of the LEM when neither of its disjuncts $A$ nor $\sim A$ would hold, as is the case with the set-theoretic and semantic paradoxes. Thus, the general use of the LEM would require a failure of the Priming Property: If $A \vee B$ is a theorem, so is either $A$ or $B$ is a theorem. However, there is always the special use of the LEM obtained by application of the Addition Rule to one of its disjuncts, which can form part of a classical re-capture.

Brady $[201 \mathrm{x}]$ sets out the two situations where the Priming Property for theorems would be expected to fail, viz. where $A$ and $B$ are schematic, as occurs in the statement of theorem-schemes, and where $A \vee B$ occurs in a subproof, the hypothesis of which may not allow the derivation of $A$ or $B$, even though it does allow that of $A \vee B$. In the first situation, individual 
instances of $A$ or $B$ must hold, such as to yield $A \vee B$, but neither of the schemes $A$ or $B$ need to hold. (We regard metacomplete logics as somewhat special in satisfying Priming, but this property may not extend to applications of such logics.) In the second situation, $A \vee B$ can be assumed or derived from an assumption without $A$ or $B$ being derived. This can be seen from the shape of the standard rule for disjunction elimination in natural deduction: If $C$ is derivable from $A$ and from $B$ then if $A \vee B$ then $C$. Here, $C$ is derivable from both $A$ and $B$, but neither $A$ nor $B$ need be derivable. Thus, Priming can fail here.

The Priming Property should hold otherwise and each usage of the LEM in the context of the reductio arguments that occur in paradox derivation and in the three diagonal arguments are indeed constant instances and are not subject to hypothesis. So, neither of the above two exceptions hold and thus the Priming Property should hold. Thus, the LEM should fail in these usages, as has been seen for the paradoxes in Brady [2006], for Cantor's diagonal argument in Brady and Rush [2008], and as will be seen for the remaining two diagonal arguments in $\S 5$ and $\S 6$. However, the usages of the LEM do differ and this will be discussed in $\S 7$.

\section{The Use of the LEM and Contraction Principles in the Derivation of Paradoxes}

Brady in [2015a] sets out the reasons why our logic MCQ of meaning containment should be used to solve the set-theoretic and semantic paradoxes. The essential reason is that each of the paradoxes are expressed as a definition, which then needs to be captured in a logical system. MCQ is seen to be ideal as a logic of meaning containment to capture meaning identity, which is what definitions represent. Brady in [2006] shows that the LEM and contraction principles such as $A \rightarrow . A \rightarrow B \Rightarrow A \rightarrow B$ (or its deductive equivalent, $A \&(A \rightarrow B) \rightarrow B)$ are used in the derivation of the paradoxes and that none of these principles are incorporated in our logic MC. (As indicated in Footnote 1, MC is a slight weakening of the logic $\mathrm{DJ}^{d}$ of Brady [2006].) As is well known, the Liar paradox can be formulated as $L \leftrightarrow \sim L$, for the Liar sentence $L$ : This very sentence $L$ is false. Similarly, for the Russell's paradox, $R \in R \leftrightarrow \sim R \in R$ holds, where $R$ is the set of all sets that are not members of themselves. As argued above in $\S 2$, the LEM plays a key role in the derivation of these paradoxes and others that are similarly based. Further, the Curry paradox, which uses the contraction rule in its derivation, can be formulated as $C \in C \leftrightarrow . C \in C \rightarrow p$. (However, our main focus will be on the use of the LEM.) Further, the theories surrounding these paradoxes have been shown in Brady [2006] to be simply consistent, based on a logic such as MCQ. 


\section{The Use of the LEM in Cantor's Diagonal Ar- gument}

We pick up on the derivation of Cantor's diagonal argument used to prove that there is no one-one correspondence between the set of natural numbers and its power set. The classical argument is set out in $\S 7$ of Brady and Rush [2008], where a one-one function $f$ from the set $\mathbb{N}$ of natural numbers to its power set $\mathcal{P}(\mathbb{N})$ is introduced and a diagonal set $D$ defined which satisfies the two conditions, $D \subseteq \mathbb{N}$ and, for all natural numbers $n \in \mathbb{N}$, $n \in D$ iff $\sim n \in f(n)$. Then, since $D \in \mathcal{P}(\mathbb{N})$, there must be an element $k$ of $\mathbb{N}$ such that $f(k)=D$. So, for this element $k, k \in D$ iff $\sim k \in f(k)$, and hence $k \in f(k)$ iff $\sim k \in f(k)$, which puts the argument into the shape $A \Rightarrow B \leftrightarrow \sim B$ mentioned above for diagonal arguments, where the $A$ is the one-one correspondence between $\mathbb{N}$ and $\mathcal{P}(\mathbb{N})$ and $B$ is the statement $k \in f(k)$, representing the membership of the element $k$ in the diagonal set $D$.

When we take the argument out of its classical context, as in Brady and Rush [2008], and raise the question as to whether the LEM holds for B or not, we see that it should fail because $f$ is not necessarily recursive since we must take into account all functions in an attempt to show that there is a one-one function at all between $\mathbb{N}$ and $\mathcal{P}(\mathbb{N})$. And, given Church's Thesis, it is only if $D$ is a recursive set, in which case $f$ would need to be recursive also, that a procedure can be determined that proves that either $k \in f(k)$ or that its negation holds, which would in turn ensure that the LEM holds. As we noted above, the LEM should not hold without support from one of its disjuncts in a constructive logic such as MC.

\section{The Use of the LEM in the Proof of Gödel's First Theorem}

The LEM impacts both the representation of negation in recursive arithmetic and the diagonal argument that constitutes the proof of Gödel's First Theorem. As we will see, the LEM will again be assumed to hold in cases where both of its disjuncts are unproven.

We start by considering the negation of the meta-language and then comparing it with that of the object system, which is based on the language of arithmetic. We will proceed by examining pertinent points from Chapter 3 on Formal Number Theory in Mendelson [2010], pp.149-226. We start with p.166-7 of Mendelson [2010], where it is stated: "Number-theoretic functions and relations are intuitive and are not bound up with any formal system". We see that this view has not changed over the years as the same statement is made in Mendelson's original edition in [1964] on p.117 as has been made in his $5^{\text {th }}$ edition in [2010] on pp.166-7. Thus, number- 
theoretic relations, and hence functions, are taken to be informal concepts within a non-formalized meta-theory, which is where such intuitive concepts would reside. However, formalization is a natural practice for elucidating any proof process by making clear what the basic logic is and the logical steps involved in any argumentation using this logic. Further, proof theory arising from such formalization is the theory of applying logical deduction to all arguments wherever they might occur, and meta-theory should be no exception to this. Thus, one should not absent oneself from proof theory even though one is dealing with intuitive arithmetical relations and functions.

Mendelson, on p.167 of [2010], goes on to define number-theoretic expressibility, into a formal theory $\mathrm{K}$ in the language of arithmetic, for such an "intuitive" relation $\mathrm{R}$ of $\mathrm{n}$ arguments thus:

1. if $R\left(k_{1}, \ldots, k_{n}\right)$ is true then $\vdash_{K} B\left(\bar{k}_{1}, \ldots, \bar{k}_{n}\right)$, and

2. if $R\left(k_{1}, \ldots, k_{n}\right)$ is false then $\vdash_{K} \sim B\left(\bar{k}_{1}, \ldots, \bar{k}_{n}\right)$,

for any natural numbers $k_{1}, \ldots, k_{n}$, where $B\left(x_{1}, \ldots, x_{n}\right)$ is some formula of $\mathrm{K}$ with free variables, $x_{1}, \ldots, x_{n}$. (Note that $\bar{k}$ is the numeral for the number $k$ and that we replace Mendelson's script letters with standard letters, letting the context differentiate their respective uses.) One would understand here that such meta-theory is assumed to be based on classical two-valued logic, evidenced by truth and falsity being taken as the only values under consideration for the relation $R$, and by its placement in a twentieth century mathematical context. Here, falsity would just be a fall-back when truth does not apply, that is, negation just represents non-truth, which would not need to be proved in any constructive way. It holds just when truth does not apply. This problem would then be projected into the object theory through the representation of the meta-theory into the object theory using the above expressibility, together with recursion and Gödel numbering, as we will see later. Further, the lack of proof theory within the meta-theory prevents any well-determined separation of truth and proof in the meta-theory of Peano arithmetic. It is a question, then, of whether this lack of separation projects into the object theory. And, it was only when Gödel's First Theorem was proved that it provided the impetus to separate proof from truth in the first place. It is only with this hindsight that one can go back and consider such a separation, but this is what we will do.

Let us consider the consequences of replacing the above truth and falsity respectively by provability and provability of the non-proof in a meta-theory based on proof, focussing instead on proof rather than truth. And, logic is about provability, as has been argued in Brady [2015], Brady [201x] and elsewhere, contrary to the above approach to the meta-theory. Then, it is not clear at all that these two are the only possibilities, since it would be possible for neither $R\left(k_{1}, \ldots, k_{n}\right)$ nor not- $R\left(k_{1}, \ldots, k_{n}\right)$ to be provable. That is, based on logic being about proof rather than truth, as was argued 
in Brady [201y], a classical meta-theory requires decidability of the object logic, as well as a sound methodology for establishing non-proof. And, it is not clear at all that arithmetic is decidable, given that classical predicate calculus is undecidable using classical processes.

Nevertheless, let us consider Mendelson's classical account of the metatheory. Under the expressiblity of $R\left(k_{1}, \ldots, k_{n}\right)$ and not- $R\left(k_{1}, \ldots, k_{n}\right)$ into $\mathrm{K}$, the two-valuedness of the relation $R$ would mean that the LEM holds which then extends to the proofs of $B\left(k_{1}, \ldots, k_{n}\right)$ and $\sim B\left(k_{1}, \ldots, k_{n}\right)$ in $\mathrm{K}$ by ensuring that either of these are provable in K. Further, in the contrapositive direction, if we assume the simple consistency of the arithmetic (as in the statement of Gödel's First Theorem) then not both $B\left(k_{1}, \ldots, k_{n}\right)$ and $\sim$ $B\left(k_{1}, \ldots, k_{n}\right)$ would be provable and, by expressibility, $R\left(k_{1}, \ldots, k_{n}\right)$ is not both true and false, establishing that they do not overlap. These are a few of the impacts of the classical meta-theory.

Taking this further, such classicality can be shown using characteristic functions. These functions are defined on p.170 of Mendelson [2010], where the characteristic function $C_{R}$, for a relation $R$ of $\mathrm{n}$ arguments, is defined such that:

$$
\begin{gathered}
C_{R}\left(x_{1}, \ldots, x_{n}\right)=0 \text { if } R\left(x_{1}, \ldots, x_{n}\right) \text { is true, and } \\
C_{R}\left(x_{1}, \ldots, x_{n}\right)=1 \text { if } R\left(x_{1}, \ldots, x_{n}\right) \text { is false. }
\end{gathered}
$$

On p.171, the following can be easily shown: $C_{n o t-R}=1-C_{R}, C_{(Q \text { or } R)}=$ $C_{Q} \times C_{R}$, and $\mathrm{C}_{(Q \text { and } R)}=C_{Q}+C_{R}-C_{Q} \times C_{R}$, where $Q$ and $R$ are relations of $n$ arguments. (This is left as an exercise by Mendelson.) We focus particularly on how this impacts upon negation. The characteristic function is set up in such a way as to embrace the classicality of the metalogic by allowing only the two values, 0 and 1, completely determined in accordance with the truth and falsity of the relation $R\left(x_{1}, \ldots, x_{n}\right)$. This can be further seen by the way the characteristic function behaves with respect to negation, disjunction and conjunction of the relations $Q$ and $R$. Negation simply changes the value, the characteristic function for the disjunction takes the (false) value 1 iff both disjuncts are false, and similarly the characteristic function for the conjunction takes the (true) value 0 iff both conjuncts are true. Thus, the characteristic functions serve to cement the classicality of the meta-logic into the object logic by capturing its truth-tables, which was surmised in the above discussion. As stated earlier, this then means that negation is just a fall-back for truth, rather than being independently established by a process of argument, this being ensured by the narrowing down of the characteristic function to the two values 1 and 0 , which do the work of the two truth-values.

Before we proceed further, we complement the notion of expressibility for relations by adding the notion of of representability of functions for any 
theory K with equality in the language of arithmetic, as follows. (See [2010], p.167.)

A number-theoretic function $\mathrm{f}$ of $\mathrm{n}$ arguments is said to be representable in $\mathrm{K}$ iff there is a formula $B\left(x_{1}, \ldots, x_{n}, y\right)$, for free variables, $x_{1}, \ldots, x_{n}, y$, such that for any natural numbers $k_{1}, \ldots, k_{n}, m$, the following hold:

1. if $f\left(k_{1}, \ldots, k_{n}\right)=m$ then $\vdash_{K} B\left(\bar{k}_{1}, \ldots, \bar{k}_{n}, m\right)$, and

2. $\vdash_{K}\left(\exists_{1} y\right) B\left(\bar{k}_{1}, \ldots, \bar{k}_{n}, y\right)$.

As in [2010], we then define strongly representable in K by replacing condition 2 by:

$$
\vdash_{K}\left(\exists_{1} y\right) B\left(x_{1}, \ldots, x_{n}, y\right) .
$$

Primitive recursion and (general) recursion for functions are defined on pp.171-2 of [2010], and this is extended to primitive recursive and (general) recursive relations on p.177 of [2010] in accordance with that of their corresponding characteristic functions. (The word 'general' is subsequently dropped, that is, 'recursion' will mean 'general recursion'.) The same sort of definition applies to sets of natural numbers. Then, on pp.177-8 of [2010], the following is proved via these characteristic functions:

If the relation $R\left(x_{1}, \ldots, x_{n}\right)$ is primitive recursive (or recursive)

then $\sim R\left(x_{1}, \ldots, x_{n}\right)$ is also primitive recursive (or recursive).

This extends to the other connectives as well. Prima facie, this result for negation seems surprising as such a result would entail the decidability of recursive arithmetic properties by interleaving the two recursions for $R$ and for $\sim R$. As we will see below, arithmetic proof is recursive and, applying this result, non-proof would also be recursive, with the decidability of arithmetic following. This then ties in with our earlier discussion.

On pp.185-8 of [2010], it is proved that every recursive function is representable in $\mathrm{S}$ and that every recursive relation is expressible in $\mathrm{S}$, where $\mathrm{S}$ is first-order Peano arithmetic, as axiomatically set out on pp.149-150 of [2010]. We then have the important result that proof in $\mathrm{K}$ is recursive, using Gödel numbering. On p.197 of [2010], it is proved that the following three relations are primitive recursive (or recursive):

- $A x(y): y$ is the Gödel number of an axiom of $\mathrm{K}$.

- $\operatorname{Prf}(y): y$ is the Gödel number of a proof in K.

- $\operatorname{Pf}(y, x): y$ is the Gödel number of a proof in $\mathrm{K}$ of a formula with Gödel number $x$. 
On p.199, a closer connection between recursion and the expressibility of relations and the representability of functions is then shown. That is, upon a modest assumption and upon the consistency of $\mathrm{S}$, a function is recursive iff it is representable in $\mathrm{S}$, and a number-theoretic relation $\mathrm{R}$ is recursive iff it is expressible in $\mathrm{S}$.

We then go on to consider the diagonal argument used to prove Gödel's First Theorem. On p.196 of [2010], the diagonal function $D(u)$ is defined as the Gödel number of $B(\bar{u})$, if $u$ is the Gödel number of a formula $B\left(x_{1}\right)$. On pp.202-209 of [2010], Mendelson goes on to prove Gödel's Incompleteness Theorem and then the Gödel-Rosser form of the Theorem, as follows. If we assume that the diagonal function $D$ is representable in a theory $\mathrm{K}$ with equality in the language of arithmetic, then the Diagonalization Lemma states that, for any formula $E\left(x_{1}\right)$ in which $x_{1}$ is the only free variable, there is a closed formula $C$ such that $\vdash_{K} C \equiv E(\ulcorner C\urcorner)$, where $\ulcorner C\urcorner$ is the Gödel number of $C$, expressed as a numeral. Since $D$ is a recursive function and is thus representable in $\mathrm{K}$, the Diagonalization Lemma holds. (Mendelson then calls this the "Fixed-Point Theorem".)

Gödel's Incompleteness Theorem is then proved in the form:

Let $\mathrm{K}$ be a theory with equality in the language of arithmetic, satisfying the three conditions: $K$ has a recursive axiom set, $\vdash_{K} \overline{0} \neq \overline{1}$, and every recursive function is representable in $\mathrm{K}$. Then:

$$
\begin{aligned}
& \text { if } \mathrm{K} \text { is (simply) consistent, then not- } \vdash_{K} G \text {, and } \\
& \text { if } \mathrm{K} \text { is } \omega \text {-consistent, then not- } \vdash_{K} \sim G,
\end{aligned}
$$

where, applying the Diagonalization Lemma, $G$ is the Gödel sentence which satisfies:

$$
\vdash_{K} G \equiv \forall x_{2} \sim P f\left(x_{2},\ulcorner G\urcorner\right)(*) .
$$

That is, $\mathrm{G}$ expresses its own unprovability.

Recall that $\mathrm{K}$ is $\omega$-consistent iff, for every formula $B(x)$, with $x$ as its only free variable, if $\vdash_{K} \sim B(\bar{n})$ for every natural number $n$, then not $\vdash_{K}(\exists x) B(x)$. We note that $\omega$-consistency of $\mathrm{K}$ implies the consistency of $\mathrm{K}$, by a lemma on p.204. However, this proof uses the (admittedly arbitrary) tautology $\sim(A \& \sim A)$, but more importantly makes the inference from non-triviality to consistency, which follows due to the presence of the rule $A \& \sim A \Rightarrow B$ in the logic. This step would fail in a good logic such as MC, which is paraconsistent. What we will then do is to assume consistency, as well as $\omega$-consistency, thus avoiding the lemma.

We now look into the details of the proof, given on pp.205-6, of Gödel's Incompleteness Theorem. There are two parts each yielding one of the two non-theorems of K, $G$ and $\sim G$, and we consider each of these derivations individually. 
The first part proves the inconsistency of $\mathrm{K}$ by a reductio argument upon the assumption of $\vdash_{K} G$. Let $r$ be the Gödel number of such a proof in $\mathrm{K}$ of $G$ and let $q$ be the Gödel number of $G$, and thus $\operatorname{Pf}(r, q)$ is a true (meta-theoretic) statement. By expressibility, $\vdash_{K} \operatorname{Pf}(r,\ulcorner G\urcorner)$, where $P f$ is the corresponding relation in K. By (*) above, $\vdash_{K} \forall x_{2} \sim P f\left(x_{2},\ulcorner G\urcorner\right)$ and hence, by instantiation, $\vdash_{K} \sim P f(r,\ulcorner G\urcorner)$, which yields a contradiction. Thus, $\mathrm{K}$ is inconsistent. By contraposition, if $\mathrm{K}$ is consistent then not$\vdash_{K} G$. However, due to the expressibility of the meta-theoretic relation $P f(r, q)$ as a numerical theorem, $\vdash_{K} P f(\bar{r},\ulcorner G\urcorner)$, the classicality of the logic enters into the argument and the inference from $\vdash_{K} G$ to the inconsistency of $\mathrm{K}$ takes the form of a classical rule. In order to contrapose this inference, the LEM and the DS enter, as discussed in $\S 2$, with the LEM applying to the provability of G and the DS applying to the inconsistency of K. If this argument was conducted in a constructive logic like MCQ, the use of the LEM for provability would require (the unlikely) decidability of $\mathrm{K}$, together with a sound methodology for determining non-proof, as indicated earlier in this section and more fully discussed in Brady [201y].

For the second part, we assume the $\omega$-consistency of $\mathrm{K}$ and $\vdash_{K} \sim G$. By applying contraposition to $(*), \vdash_{K}\left(\exists x_{2}\right) P f\left(x_{2},\ulcorner G\urcorner\right)$. (Note that, if this was conducted in MCQ, the bi-conditional in $\left(^{*}\right)$ would translate as a co-entailment, as occurs in other diagonal arguments, and this readily contraposes in MCQ.) Since $\vdash_{K} \sim G$, together with $\omega$-consistency implying consistency, not- $\vdash_{K} G$ follows, and hence there is no natural number $\mathrm{n}$ which is the Gödel number of a proof of $G$ in K. That is, $\operatorname{Pf}(n, q)$ is false for every natural number $n$. By expressibility, $\vdash_{K} \sim P f(\bar{n},\ulcorner G\urcorner)$, for every natural number $n$ and, by $\omega$-consistency, not- $\vdash_{K}\left(\exists x_{2}\right) P f\left(x_{2},\ulcorner G\urcorner\right)$, contradicting $\vdash_{K}\left(\exists x_{2}\right) P f\left(x_{2},\ulcorner G\urcorner\right)$ above. Thus, the meta-theory of $\mathrm{K}$ is inconsistent. Assuming $\omega$-consistency, by contraposition, if the meta-theory of $\mathrm{K}$ is consistent then not- $\vdash_{K} \sim G$. As for the first part, the use of expressibility does introduce classical logic and the inference involved in this derivation is also a classical rule. Let us look more closely at the structure of the general argument, that is:

If $\mathrm{K}$ is $\omega$-consistent (and hence $\mathrm{K}$ is consistent by classical inference) and $\vdash_{K} \sim G$ then the meta-theory of $\mathrm{K}$ is inconsistent.

Let us consider the argument in MCQ. This would then contrapose into:

If the meta-theory of $\mathrm{K}$ is consistent then not $-\vdash_{K} \sim G$ or $\mathrm{K}$ is $\omega$ inconsistent or $\mathrm{K}$ is inconsistent, with the use of the LEM and the DS. Further, by adding the $\omega$-consistency and the consistency of $\mathrm{K}$ to the consistency of the meta-theory of $\mathrm{K}$ as premises, then not $-\vdash_{K} \sim G$ follows, by applying the DS twice.

So, we can also see that the LEM and the DS are applied to the metatheoretic statements of arithmetic to put Gödel's Incompleteness Theorem 
into its familiar form. Again, if a logic such as MCQ is used, the decidability of $\mathrm{K}$ and a sound methodology for establishing non-proof would be required to achieve this.

Gödel and Rosser go on to strengthen Gödel's Incompleteness Theorem by replacing $\omega$-consistency by consistency, using a Rosser sentence $\mathrm{R}$ and adding two obvious conditions on $\mathrm{K}$. Although the argument is more complicated, a similar two-part structure is used. That is, if $\vdash_{K} R$ then it is proved that $\mathrm{K}$ is inconsistent, and if $\vdash_{K} \sim R$ then $\vdash_{K} R$, which yields a contradiction. So, if $\mathrm{K}$ is consistent then neither $\vdash_{K} R$ nor $\vdash_{K} \sim R$, where $R$ is the Rosser sentence for $\mathrm{K}$, as set out in detail on p.207. The first part uses the same sort of expressibility as for the first part of Gödel's Incompleteness Theorem, once for the Gödel number of a proof of $\mathrm{R}$ in $\mathrm{K}$ and once more for the Gödel number of a proof of $\sim R$ in $\mathrm{K}$. The second part uses the same sort of expressibility as for the second part of Gödel's Incompleteness Theorem, but also uses a number of instances of the Deduction Theorem within the axiomatized system $\mathrm{K}$. As stated above in $\S 2$, such uses of the Deduction Theorem are deductively equivalent to the LEM. We will discuss such usages of the LEM in $\S 7$.

The use of the LEM, the DS and classicality in general in Gödel's proofs are significantly different from that of the other reductio arguments discussed in this paper.

\section{The Use of the LEM in the Proof of the Unde- cidability of Turing's Halting Problem}

A reductio argument in the spirit of Cantor's diagonal argument is used in the standard proof of the undecidability of Turing's halting problem, and this of course implicitly requires the LEM. We follow Papadimitriou in [1994], pp.57-65, which introduces, in his chapter on undecidability, the universal Turing machine that is construed broadly enough to be able to apply to itself. That is, a Turing machine $\mathrm{M}$ can have as its input a Turing machine that could indeed be itself. A Turing machine operates on a string, moving the cursor to the right on accepting an input and halting when it can accept no further input.

We start by defining the halting set $H$ of pairs $(M ; x)$, consisting of a Turing machine $M$ together with an input $x$, such that the Turing machine halts on that input. For the sake of the reductio argument, let $M_{H}$ be the Turing machine that decides $H$. That is, $M_{H}$ accepts as input precisely the members of $H$, rejecting those that are not in $H$. We define the diagonal machine $D(M)$, for a machine $M$, so that when $M_{H}$ accepts as input a Turing machine $M$ with itself $M$ as input, that is $M_{H}(M ; M), D(M)$ 's state is the opposite of that of $M_{H}(M ; M)$. That is, if $M_{H}(M ; M)$ halts then $D(M)$ moves the cursor to the right and, vice versa, if $M_{H}(M ; M)$ 
moves the cursor to the right then $D(M)$ halts.

We then raise the question of what happens when $D$ is applied to itself. If $D(D)$ moves the cursor to the right then $M_{H}$ accepts $(D ; D)$ as input, in which case $(D ; D) \in H$ and then $D(D)$ halts. On the other hand, if $D(D)$ halts, $M_{H}$ rejects the input $(D ; D)$, it is not the case that $(D ; D) \in H$, and then $D(D)$ moves the cursor to the right. This argument has the shape: if $M_{H}$ is a Turing machine that decides the set $H$ then $D(D)$ halts iff $D(D)$ moves the cursor to the right, that is, $D(D)$ does not halt. That is, as for Cantor's diagonal argument, it takes the shape: $A \Rightarrow B \leftrightarrow \sim B$. So, by use of the LEM on $B$, as described above in $\S 2$, this creates the contradiction $B \& \sim$ $B$, that is, that $D(D)$ both halts and does not halt, upon the assumption that $\mathrm{H}$ is decidable. By the classical reductio argument, there is no Turing machine $M_{H}$ that decides $H$, and so the halting problem is undecidable. This then gives rise to undecidability proofs in general by projecting the substance of universal Turing machines into other areas.

However, we can also raise the question of whether the LEM should hold for the halting or otherwise of $D(D)$. As with Cantor's diagonal argument, LEM should be supported through one of its disjuncts, $D(D)$ halts or $D(D)$ moves the cursor to the right. Neither of these have the required support as they both would need some recursive deductive process for the argument to their respective conclusions to take shape and this has not been established, especially in light of the classical undecidability result. We will discuss this usage of the LEM in a more general context in the next section.

\section{In Conclusion}

We start by delineating four different usages of the LEM amongst the above cases. The case of the set-theoretic and semantic paradoxes provides the first usage, where the LEM produces an outright contradiction $B \& \sim B$ from an equivalence of the form $B \leftrightarrow \sim B$. This paradox (generally, the Liar and Russell's Paradoxes) is then solved by choosing one's logic in accordance with meaning containment and its use in capturing definitions used in setting up the paradoxes. (See Brady [2015a] for the detailed discussion of this point, plus a broader range of paradoxes.) It has been shown in Brady [2006] that, in a constructive logic such as MCQ without the LEM, such contradictions are not derivable, and that there are no deductive processes that establish the $B$ or the $\sim B$ of the $B \vee \sim B$, for the above $B$.

The cases of Cantor's diagonal argument of $\S 4$ and the diagonal argument concerning Turing machines in $\S 6$ provide the second usage, where the LEM is still used to create a contradiction $B \& \sim B$ from a conclusion of the form $B \leftrightarrow \sim B$, but upon an assumption, with the reductio argument then used to deny the assumption. It is this reductio argument, which employs rule-contraposition, which uses the LEM, as well as the DS, from classi- 
cal logic. Also, given that MCQ is weaker than classical, any classically uncountable set cannot be shown to be countable. However, classically undecidable sets may be shown to be decidable in a weaker logic. For example, the quantified logic LDWQ is decidable, whilst the classical predicate calculus is not. See Brady [1996] and [1996a]. ${ }^{6}$ This would leave the classically uncountable sets and may leave undecidable sets undetermined using a logic such as MCQ. Intuitively, this would be expected with such a constructive logic, as a negative result such as uncountability or undecidability would need a constructive argument to its conclusion. These are major changes to classical mathematics, requiring a complete re-think and re-write for the fields of infinite cardinals and of undecidability.

The case of Gödel's Incompleteness Theorem provides the third usage, where the LEM and the DS are the key ingredients used, in addition to MCQ, in setting up the classical meta-theory of Peano Arithmetic. This metatheory is then expressed (and represented) within the formal object theory of arithmetic through the use of the two classical rules, one for truth and one for falsity for meta-theoretic relations. (It is clear that such expressibility goes beyond meaning containment and cannot be represented by an ' $\rightarrow$ ' in MCQ. As truth-preservation is still maintained, we would use the rule ' $\Rightarrow$ '.) As argued above, this presupposes the decidability of Peano arithmetic, which is dubious. (The author is not aware of any such result in the literature.) Gödel's Incompleteness Theorem also uses contraposition, which embodies the second usage of the LEM (and the DS).

The case of the Gödel-Rosser Incompleteness Theorem provides the fourth usage of the LEM in that it also makes use of the Deduction Theorem, applied in the object language, to create the more complex Rosser sentence $R$ for K. Some of the components of the Rosser sentence were obtained by expressibility, which is expressed as a classical rule, making the Deduction Theorem essential in creating an inferential formula. As we saw in $\S 2$, the Deduction Theorem is deductively equivalent to the LEM, which is then linked to the third usage. Such usage of the LEM is within the axiomatic system that may or may not be justified through one of its disjuncts. This leads us to the next point.

Where the LEM does also enter is in the contrast between the provability of the LEM in classical logic and the unprovability of $G$ and of $\sim G$, for the Gödel sentence $G$. That is, $G \vee \sim G$ would fail the Priming Property, and it has been argued in Brady [201x] and also in $\S 2$ that the Priming Property ought to hold in this case and hence that the logic of arithmetic should exclude the LEM. Such a logic is our logic MCQ of meaning containment, a slight immaterial weakening of which is used in Brady [2012] to develop simply consistent arithmetic, up to the point of including primitive recursion. One should note that due to MCQ being weaker than classical, the two unprovabilities of $G$ and $\sim G$ would still apply, subject of course to the consistency of the classical Peano arithmetic. 
We will just comment on the DS, as it is usually justified through a simple consistency proof of the system, which is not likely to be classically available due to Gödel's Second Theorem and due to the complexity of the systems involved. However, Brady [2012] does show that the DS is an admissible rule that is then added as a primitive rule. The general use of the LEM obviously fails in a constructive logic and so our primary focus is on it, rather than the DS that can be achieved through consistency.

We still need to address the question: "Why do we need to dismantle classical mathematics?" Whilst classical mathematics is a beautiful system and will always be studied as such, there is a need to focus our formal systems on concepts and their clarification rather than merely studying formal systems for their own sake as Hilbert did. (See Brady [201y] for a discussion of this point.) So, we need to focus our attention on the concepts that are used to guide logical systems, i.e. meaning and truth, and the concepts of mathematics, such as the initial concepts of set and number.

Our logic MCQ captures meaning and truth inferentially through its ' $\rightarrow$ '-connective, representing meaning containment, and its rule- $\Rightarrow$ ', which satisfies truth-preservation properties. This system of logic captures these concepts as best as we are able and there could be subsequent tweaking of the system, but it still represents a genuine effort to achieve this outcome. The concept of information has been more recently used as a guide to determine some relevant logics, which can be seen in Bimbo [2016], a volume on information-based logics. However, in Brady [2016] within this volume, it is argued that information is just true logical content, where such content can be regarded as an analytic closure, appropriately based on the logic MCQ. The conclusion here is that one should just focus on the logic MCQ in any case and this does cast classical logic aside as meaning is not captured there whilst it just focuses on truth and falsity alone, with all its well-known problems of relevance. This then means that classical mathematics should be replaced by a mathematics based on a logic such as MCQ, which, as this paper shows, does involve a considerable dismantling of some of its key concepts such as undecidability and uncountability, together with a re-writing of Godel's proof of incompleteness.

We finally return to Routley's comment: "Gödel's (First) Theorem would not be provable using a decent logic". We take it that Routley is referring to the derivation of the Gödel's Theorem using a weaker logic without being specific. However, as pointed out above, Gödel's (First) Theorem is achievable with a weaker logic, subject to the assumption of classical consistency. Nevertheless, Routley was thinking of working the whole proof through in a weaker logic and this paper does not do this in anywhere near fullness, but it is hoped that it would provide guidance to the key issues to be dealt with in such a process. 


\section{Notes}

1 Just to recap, in Brady [2006], the $\operatorname{logic} \mathrm{DJ}^{d} \mathrm{Q}$ was introduced and argued for as a logic of meaning containment. However, in response to Restall's review of the book in [2007], there was seen to be a need to drop the distribution axiom, $A \&(B \vee C) \rightarrow(A \& B) \vee(A \& C)$. This was indeed done in Brady and Meinander [2013], where the sentential and existential rule-forms of distribution were retained. The reason was that the sentential distribution, $A \&(B \vee C) \rightarrow(A \& B) \vee(A \& C)$, is not an example of meaning containment, and this extends to the quantified forms as well. The final system was then referred to as MCQ, with just MC for the sentential logic.

2 The term 'metacomplete' was introduced by Meyer in [1976] for logics without negation that satisfy standard truth-conditions for conjunction, disjunction, and the two quantifiers, together with its provability as well as the truth-condition embracing Modus Ponens for implication. Indeed, metavaluations have truth-conditions that include provability as well as standard truth-properties to inductively capture the connectives and quantifiers of a logic. Meyer's definition was expanded to include logics with negation by Slaney in [1984] and [1987], who distinguished M1- and M2-logics, according to whether there are no negated entailment theorems or whether negated entailments satisfy the standard truth-condition. Once soundness and completeness are proved for a metavaluation, the logic is deemed to be 'metacomplete'. Thus, one should note that truth in this context amounts to provability and that the methodology employed here is proof theory and not semantics. For a full account of metavaluations, see Brady [2107].

${ }^{3}$ Deductive equivalence is a general term that applies to deductions in both directions between two theorems or between a theorem and a derived rule or between two derived rules. In the process of such deduction, uniform substitution upon theorems can be used along with the usual usage of theorems and derived rules of the logic. Thus, a two-way derived rule, constituted as derived rules in both directions, is a special case of a deductive equivalence, as one cannot use uniform substitution in performing the derivation of a derived rule.

One should also note here that $A \rightarrow \sim A \Rightarrow \sim A$ is a form of contraction as can be seen from its left-handed Gentzen rule-form: $A: A / A$, where $A: B$ is defined as $\sim(A \rightarrow \sim B)$, an intensional conjunction called 'co-tenability' in Anderson and Belnap [1975], pp.344-6. For this style of Gentzen system, see Brady [2006], pp.110-140.

${ }^{4}$ One should note that, for intuitionist logic, $B \& \sim B$ can be derived from $B \leftrightarrow \sim B$ by using its theorem, $B \rightarrow \sim B \rightarrow \sim B$, together with $\sim B \rightarrow B$. Nevertheless, in a logic such as MC with all the De Morgan Laws, $B \vee \sim B$ and the rule-form, $B \rightarrow \sim B \Rightarrow \sim B$, are deductively equivalent, shown in 
particular by using the non-intuitionistic $\sim(B \& \sim B) \rightarrow B \vee \sim B$, and it is this rule-form that does the essential work in the intuitionist derivation.

${ }^{5}$ Conjunctive Syllogism, $(A \rightarrow B) \&(B \rightarrow C) \rightarrow . A \rightarrow C$, is also an example of a contraction principle, but this has been supported as a meaning containment and it can be incorporated in proofs of the consistency of naïve set theory and naïve truth theory, as shown in Brady [2006].

6 This difference between uncountability and undecidability is due to the fact that countability is a single one-sided process which matches a set with the set of natural numbers, whilst decidability requires both proof and non-proof to be determined by some effective means.

\section{References}

ANDERSON, A.R. and BELNAP, N.D., Jr.

[1975]: Entailment. The Logic of Relevance and Necessity, Vol.1, Princeton, U.P., Princeton.

BIMBO, K.

[2016]: (Ed.) J. Michael Dunn on Information Based Logics, Springer, Switzerland.

BRADY, R.T.

[1984]: "Natural Deduction Systems for Some Quantified Relevant Logics", Logique et Analyse, Vol. 27, pp.355-377.

[1996]: "Gentzenizations of Relevant Logics Without Distribution - I", Journal of Symbolic Logic, Vol. 61, pp. 353-378.

[1996a]: "Gentzenizations of Relevant Logics Without Distribution II", Journal of Symbolic Logic, Vol. 61, pp. 379-401.

[2003]: Relevant Logics and their Rivals, Vol.2, editor and contributor, Ashgate, Aldershot.

[2006]: Universal Logic, CSLI Publs, Stanford.

[2012]: "The Consistency of Arithmetic, Based on a Logic of Meaning Containment", Logique et Analyse, Vol.55, pp.353-383.

[2015]: "Logic - The Big Picture", in New Directions in Paraconsistent Logic, ed. by Jean-Yves Beziau, Mihir Chakraborty and Soma Dutta, Springer, New Delhi, pp. 353-373. 
[2015a]: "The Use of Definitions and their Logical Representation in Paradox Derivation", presented to the $5^{\text {th }}$ World Congress on Universal Logic, University of Istanbul, Istanbul, Turkey, June 25-30, forthcoming in a special issue of Synthese on Substructural Approaches to Paradox, ed. by Elia Zardini.

[2016]: "Comparing Contents with Information", in J. Michael Dunn on Information Based Logics, ed. by Katalin Bimbo, Springer, Switzerland, pp.147-159.

[2017]: "Metavaluations", The Bulletin of Symbolic Logic, Vol.23, pp.296323.

[201x]: "On the Law of Excluded Middle", forthcoming in a re-publication of Routley, R., Exploring Meinong's Jungle and Beyond, ed. by Maureen Eckert, Springer.

[201y]: "The Number of Logical Values", presented to the Logic Seminar, Uni. of Melbourne, March 2017, forthcoming in Graham Priest on Dialetheism and Paraconsistency.

BRADY, R.T. and MEINANDER, A.

[2013] "Distribution in the Logic of Meaning Containment and in Quantum Mechanics" in Paraconsistency: Logic and Applications, ed. by Koji Tanaka, Francesco Berto, Edwin Mares and Francesco Paoli, Springer Publishing, Dordrecht, 2013, pp.223-255.

BRADY, R.T. and RUSH, P.

[2008]: "What is Wrong with Cantor's Diagonal Argument?", Logique et Analyse, Vol.51, pp.185-219.

KAMIDE, N. and WANSING, H.

[2015]: Proof Theory of N4-related Paraconsistent Logics, College Publications, London.

MENDELSON, E.

[1964]: Introduction to Mathematical Logic, 1st edn, Van Nostrand, Princeton, New Jersey.

[2010]: Introduction to Mathematical Logic, $5^{\text {th }}$ edn, CRC Press, Boca Raton, Florida. 


\section{MEYER, R.K.}

[1976]: "Metacompleteness", Notre Dame Journal of Formal Logic, Vol. 17, pp. 501-516.

ODINTSOV, S. P.

[2008]: Constructive Negations and Paraconsistency, Springer.

PAPADIMITRIOU, C.H.

[1994]: Computational Complexity, Addison Wesley Longman, Reading, Massachusetts.

RESTALL, G.

[2007]: Review of R.T. Brady, Universal Logic, in Bulletin of Symbolic Logic, Vol. 13, pp. 544-547.

SLANEY, J.K.

[1984]: "A Metacompleteness Theorem for Contraction-free Relevant Logics", Studia Logica, Vol. 43, pp. 159-168.

[1987]: "Reduced Models for Relevant Logics without WI", Notre Dame Journal of Formal Logic, Vol. 28, pp. 395-407. 